\title{
Construção e validação de um instrumento de suporte escolar e parental na formação esportiva do adolescente
}

http://dx.doi.org/10.11606/1807-5509202000010033

\author{
Erasmo Braz SANTOS* \\ Layla Maria Campos ABURACHID* \\ Christianne de Faria COELHO-RAVAGNANI" \\ Fabricio Cesar de Paula RAVAGNANI, ${ }^{* * * *}$ \\ *Faculdade de Educa- \\ ção Física, Universi- \\ dade Federal de Mato \\ Grosso, Cuiabá, MT, \\ Brasil. \\ ${ }^{* *}$ Instituto Federal de \\ Ciência e Tecnologia \\ de Mato Grosso, Cuia- \\ bá, MT, Brasil.
}

\section{Resumo}

Objetivou-se construir e validar um questionário para verificar a contribuição da escola e da família na formação esportiva do adolescente. Participaram da pesquisa 295 adolescentes praticantes de esportes coletivos e individuais. Foram realizadas as seguintes etapas para validação do instrumento: validação teórica, que consistiu na construção do instrumento e na aplicação do questionário piloto; e validação empírico analítica, que consistiu na aplicação do questionário, verificação da confiabilidade, verificação da adequabilidade, aplicação da análise fatorial exploratória e na modelagem final do questionário. As análises de confiabilidade, adequabilidade e fatorial exploratória realizadas para identificar evidências de validade de construto do questionário apresentaram ajustes aceitáveis e resultados que atenderam as recomendações da literatura. 0 modelo final do questionário abarcou 21 itens dos 39 anteriores analisados e foram distribuídos em duas dimensões: Escola com nove itens divididos em fatores denominados de (1) desenvolvimento esportivo (itens 1, 2, 3, 5 e 6) e (2) desempenho escolar com (itens 7, 8, 11 e 12); e Família com 12 itens divididos em fatores denominados de (1) aspectos financeiros (itens 1, 2 e 3), (2) aspectos motivacionais (itens 11, 12 e 13), (3) interação esportiva (7, 8 e 9) e (4) acompanhamento esportivo (itens 16, 19 e 21). Conclui-se que o questionário apresentou níveis satisfatórios de fidedignidade e consistência interna com evidências de validade de construto para verificação do apoio que a escola e família concentram na formação esportiva do adolescente.

Palavras-chave: Validação; Esporte; Adolescente; Apoio Escolar; Apoio Parental.

\section{Introdução}

A escola e a família são apontadas como os principais suportes do desenvolvimento esportivo dos adolescentes, propiciando, por exemplo, as condições adequadas de infraestrutura, financeira, educacional e motivacional para que os mesmos se beneficiem da prática esportiva ${ }^{1,2}$. Os benefícios oriundos do esporte nessa fase da vida são variados e envolvem a competência física, aceitação e aprovação social, afiliação, interação com a família e professor ${ }^{3}$, desenvolvimento cognitivo e melhora da aptidão física ${ }^{4,5}$. Observa-se ainda, que o adolescente envolvido com o esporte se mantém ativo fisicamente, e tende transportar esse hábito para a vida adulta ${ }^{6}$. No campo da inclusão social, o esporte tem sido utilizado para atrair crianças e adolescentes em estado de vulnerabilidade social, afastando-os de riscos como a violência, o uso de drogas, analfabetismo, trabalho infantil e prostituição que causam mal à saúde e ao desenvolvimento pleno ${ }^{7-9}$.

Estudos têm sido realizados com fins de investigar as contribuições da escola ou da família na formação esportiva do adolescente. Em um estudo clássico ${ }^{2} \mathrm{com}$ talentos de diversas áreas constatou-se dentre outras variáveis que o aporte financeiro principalmente na infância e na adolescência tem sido quase que exclusivo da família. Em outro estudo examinando o impacto econômico nas famílias australianas, constatou-se que o gasto anual das famílias com crianças e adolescentes envolvidas no esporte girava entre 500 a mil dólares anuais ${ }^{10}$. Nos Estados Unidos, adolescentes praticantes do hóquei geravam para suas famílias custos anuais 
entre cinco a 16 mil dólares ${ }^{11}$. Em outro exemplo vindo do Canadá, adolescentes promissores no esporte geravam anualmente custos entre 10 a 20 mil dólares anuais para suas famílias ${ }^{12}$. Em estudo com nadadores brasileiros constatou-se que no que tange ao aspecto financeiro o apoio parental foi considerado como "pilar fundamental" para a maioria dos atletas estudados ${ }^{13}$. Mesma constatação foi observada no tratamento com atletas de ginástica artística no qual pais além do apoio financeiro às suas filhas cotizavam-se e ajudavam a manter os salários dos professores ${ }^{14}$. Num contraponto, um estudo realizado com atletas de basquetebol de base foi observado que sem o apoio financeiro da família há grandes chances de abandono do esporte ${ }^{15}$.

Há uma gama de estudos que criaram instrumentos quantitativos com fins de verificar os suportes parental e escolar no esporte. Nota-se porém que, nesses instrumentos foi dada grande ênfase para a participação familiar em detrimento da escolar ${ }^{16-19}$. Outros focam em lembranças de práticas parentais ${ }^{20}$, aspectos psicológicos $^{21,22}$, motivacionais ${ }^{23,24}$, focados em talentos esportivos $^{25}$, direcionados a um esporte específico ${ }^{26} \mathrm{e}$ atletas de alto rendimento ${ }^{19}$. O apoio escolar no que concerne as condiçōes das práticas esportivas também tem sido estudado, porém tendo como foco os gestores escolares $^{27}$.

Percebe-se que estudos e instrumentos são incipientes no que se refere aos adolescentes "comuns" ou não talentos, ou seja, aqueles que não reúnem condiçõos de excepcionalidades físicas e motoras de serem considerados talentos esportivos ou mesmo de se tornarem atletas de alto rendimento. Aplicar os instrumentos existentes em população de adolescentes que não reúnem essas condições (física e motora) produziria viés de informação acerca do suporte escolar e parental na sua formação esportiva, uma vez que esses instrumentos abordam questóes voltadas para os ambientes de preparação, competição e resultados que são comuns ao alto-rendimento, daí entende-se que este estudo por ora seja original.

A participação dos adolescentes talentos ou não talentos é relevante no processo de formação esportiva.
Esta é definida como um período no qual se opta pelo direcionamento da vida esportiva que objetiva a adequação de processos e os meios a serem seguidos para contribuírem na delimitação, conformação da personalidade e na busca de um referencial da cultura de movimento. No caso específico do adolescente "comum" deve-se conduzir para o usufruto do lazer e da saúde ${ }^{28}$.

O suporte escolar e familiar neste processo é relevante, pois, na maioria do seu tempo, o adolescente está sob os cuidados da escola e da família. Há que se relatar que essas duas dimensões (que são bidimensionais em relação às distribuiçōes de responsabilidades), irão influenciar sobremaneira o adolescente nessa fase da vida, na qual ocorrem concomitantemente transformações de ordem fisiológica, motora e psicológica.

Preconiza-se, por meio da psicometria, ser possível construir um instrumento quantitativo para a verificação desse suporte escolar e parental, estabelecendo evidências de validade de construto, confiabilidade e validação teórica ${ }^{29}$. A validade pode ser conceituada como a capacidade de um instrumento em medir com precisão o conceito em estudo, ou seja, avaliar aquilo que ele se propóe ${ }^{30}$. Por sua vez, a validade de construto verifica um atributo ou qualidade que não é operacionalmente definido ${ }^{30}$. Para verificação das evidências de validade de construto pode ser utilizada a análise fatorial exploratória, a partir dos padrões de correlações em instrumentos psicométricos. A confiabilidade verifica o grau em que um conjunto de indicadores de uma variável latente (construto) é consistente em suas mensurações. Ela pode ser verificada através da consistência interna pelo alfa de Cronbach, que vão refletir o nível de correlação dos itens de um questionário ${ }^{31}$.

Sendo assim, a pesquisa em voga teve como diferencial dos instrumentos existentes e validados o objetivo de construir e validar um questionário para verificar o suporte escolar e parental na formação esportiva do adolescente, apoiando-se no modelo de elaboração instrumental para desenvolvimento e validação de testes psicométricos.

\section{Método}

\section{Participantes}

Esta pesquisa foi baseada no modelo de elaboração instrumental psicológico para validação de instrumentos psicométricos ${ }^{29}$.

Participaram do estudo no que tange a validação de conteúdo (análise consensual) sete peritos com formação em educação física, com no mínimo 10 anos de experiência na área esportiva e todos com doutorado. Para a evidência de construto a amostra 
foi composta por 295 adolescentes (180 do sexo masculino e 115 do sexo feminino) com média de idade de 13,71 $\pm 1,14$ anos, todos praticantes de esportes na própria instituição escolar que estavam matriculados. A seleção da amostra adotou o preceito de aleatória intencional ${ }^{29}$.

No que se refere ao número de observações por item, ficou estabelecida uma relação de 7,56 observaçôes por item atingindo critérios adequados da literatura especializada ${ }^{29}$, demonstrando que o tamanho da amostra de 295 participantes estava adequado para a realização do processo de validação.

Os critérios de inclusão para a amostra de validação do questionário foram: ser adolescente na faixa etária de 12 a 17 anos, praticante de esportes coletivo ou individual na escola, de forma sistemática e com assiduidade. Os critérios de exclusão foram: ser federado e questionários com itens não respondidos.

O estudo foi aprovado pelo Comitê de Ética de Pesquisa com Seres Humanos da Universidade Federal de Mato Grosso sob o n. 787.452. Os participantes deste estudo, bem como seus responsáveis e as instituições promotoras dos jogos assinaram o Termo de Consentimento Livre e Esclarecido.

\section{Procedimentos}

Seguindo os procedimentos do modelo teórico adotado, na etapa de validação teórica e experimental ${ }^{29}$, para a construção e validação do instrumento realizou-se leitura acerca do construto suporte escolar e parental que envolvia o adolescente em relação ao esporte. Após a leitura, foi definido que escola e família devido às finalidades diferenciadas em relação ao adolescente formariam dimensōes diferentes estabelecendo assim, uma bidimensionalidade do instrumento.

$\mathrm{Na}$ dimensão Escola o suporte na formação esportiva do adolescente referiu-se ao que esta dimensão propõe para o desenvolvimento no que tange aos fatores de: infraestrutura, desempenho escolar, suporte pedagógico, reconhecimento, incentivo e motivação.

$\mathrm{Na}$ dimensão família o suporte à formação esportiva do adolescente relacionou-se com os fatores: financeiro, motivacional, educacional, relação familiar, amotivação, saúde, interação esportiva e acompanhamento esportivo.

Dando sequência ao processo de construção do instrumento, foi realizada busca na literatura especializada ${ }^{13-17,19,20,24,27}$, com o objetivo de elaborar itens para as dimensões escolar e familiar quanto à verificação do apoio na formação esportiva do adolescente. Foram elaborados inicialmente 63 itens e utilizada escala likert ${ }^{32}$ com quatro níveis de respostas sendo: (1) nunca, (2) às vezes, (3) frequentemente e (4) sempre. Na construção e elaboração dos itens foi seguido o preceito de que se deva ter o triplo de itens para que seja assegurado no final um terço deles ${ }^{29}$. Após a construção, o questionário foi submetido a um corpo de profissionais de educação física, para a realização da análise consensual ${ }^{33}$.

Ainda na etapa de validação teórica e experimental, aplicou-se um estudo piloto com 32 adolescentes com características similares da amostra. A finalidade desta aplicação deu-se para verificar a aceitabilidade, a clareza, o entendimento e a organização estética do questionário (esta última com via da promoção da economicidade). O tempo médio que cada adolescente levou para responder o questionário foi de 15 minutos. Salienta-se que o estudo piloto foi realizado por um único pesquisador. Com o estudo piloto, foram realizados apenas dois ajustes no questionário: a diminuição do tamanho da fonte para que o questionário coubesse em apenas uma folha (frente e verso) e a revisão da semântica.

Partindo para a segunda parte do modelo teórico adotado (validação empírico-analítica) ${ }^{29}$ no qual o pesquisador deve planejar e realizar a coleta de dados, os participantes da pesquisa foram abordados durante a realização do X Jogos Escolares Matogrossenses, realizado na cidade de Tangará da Serra-MT no mês de agosto de 2014 e responderam ao questionário auto-administrado em momentos de descanso de jogo, principalmente no alojamento, evitando assim, que o estresse gerado pelo mesmo interferisse nas respostas.

\section{Análise dos dados}

Para a caracterização da amostra utilizou-se a análise descritiva dos dados por meio de porcentagem, média e desvio padrão. Durante o processo analítico gerou-se uma matriz de correlação e foi determinada com base nessa matriz a adequabilidade dos dados com os testes Kaiser-Meyer-Olkin-KMO (>,050) e Esfericidade de Bartlett $(\mathrm{p} \leq 0,05)^{34}$. Estando a amostra adequada, partiu-se para a realização da Análise Fatorial Exploratória-AFE ${ }^{34,35}$ verificando-se as comunalidades $(\geq 0,40)^{36}$, que é a representação da variância explicada de acordo com as correlaçốes de uma variável com outras da análise $e^{36}$. Determinouse a extração de fatores. O método de componentes principais é recomendado na literatura ${ }^{34}$ quando 
a intenção é a redução de dados para a obtenção de um número mínimo de fatores e explicarem o máximo de variância. A rotação dos fatores pode ser ortogonal ou oblíqua. $\mathrm{Na}$ ortogonal assume-se que os fatores são independentes e não tem relação entre $\mathrm{si}^{37}$. Dentre os métodos de rotação ortogonais existentes o mais comumente utilizado é o varimax, por tentar maximizar a dispersão das cargas dentro dos fatores e agregar um número menor de variáveis em cada fator ${ }^{38}$. Após a rotação realizada gerou-se o eigenvalue $(\geq 1)$ que é um método utilizado para definir o número adequado de fatores, no qual se

\section{Resultados}

Dos participantes $62,3 \%$ estudavam em escola pública, $84 \%$ cursavam o ensino fundamental, $68 \%$ participavam de esportes coletivos e 53,6\% eram oriundos das classes econômicas familiares B1-B2 ${ }^{39}$.

Após a realização da análise consensual algumas reformulações e extrações de itens foram propostas e chegou-se ao modelo do questionário descartandose 24 itens e permanecendo 39, distribuídos em 12 para a dimensão Escola e 27 para dimensão Família. Todos os itens mantiveram um percentual de $\geq 80 \%$ de concordância entre os peritos ${ }^{40}$.

A partir da definição dos dados que estruturaram o questionário realizou-se os procedimentos necessários para a verificação da consistência interna (confiabilidade). No que se refere à dimensão Escola o Alfa de Conbrach geral verificado foi de 0,579 com todos os itens originais incluídos. No entanto, observou-se que o item 4 apresentou desvio padrão destoante dos demais, interferindo no valor final do alfa da dimensão. Foi promovida a purificação da escala com a extração do item e o alfa se elevou para 0,68. A dimensão Escola passou a conter 11 itens para posterior análise fatorial. Na dimensão Família o Alfa de Conbrach foi de 0,79, considerando assim, que ambas as dimensóes tem confiabilidade adequada.

A matriz de correlação realizada em seguida para a dimensão Escola passou por duas tentativas. $\mathrm{Na}$ primeira, os resultados foram: teste $\operatorname{KMO}(0,71)$ e teste de Esfericidade de Bartlett $\left(\mathrm{X}^{2}=662,50 ; \mathrm{df}=\right.$ $55 ; \mathrm{p}=0,000)$, comprovando a adequação dos dados para realização da AFE.

Foram rodados na sequência os valores das comunalidades dos itens pelo método de extração de componentes principais. Observou-se que os 11 itens foram habilitados para a próxima etapa da sugere que devem ser extraído apenas fatores com eigenvalue $(\geq 1)^{35}$. O scree plot auxilia na extração de fatores, pois gera um gráfico para análise ${ }^{35}$. Após a rotação foram determinadas as cargas fatoriais $(\geq 0,40)$ dos itens em seus respectivos fatores ${ }^{34}$. Itens que apresentaram cargas cruzadas, ou seja, cargas altas em dois fatores concomitantemente foram rechaçados. A confiabilidade foi verificada através do método comumente utilizado da consistência interna por meio do Alfa de Cronbach $\alpha(\geq 0,60)^{34}$. Os dados obtidos foram analisados com o auxílio do software SPSS 20 (Statistical Package for Social Science).
AFE, que foi a extração de fatores através da regra do autovalor $(\geq 1)$ (eigenvalue) e a percentagem da variância. Foi observado um modelo com três fatores, extraídos sendo 1 (2,7; 21,5\%), 2 (2,0;20,9\%) e $3(1,3 ; 13,9 \%)$. Esse modelo ficou adequado e conseguiu explicando 56,3\% da variância acumulada na dimensão Escola.

Dando prosseguimento a AFE a próxima etapa foi a verificação da adequada distribuição da carga fatorial de cada um dos itens entre os distintos fatores que compõem o construto. Após a rotação ortogonal varimax, o fator três carregou apenas dois itens com cargas fatoriais aceitáveis, sendo nove $(0,81)$ e 10 $(0,87)$. No intuito de construir um modelo mais robusto, os itens foram descartados da análise, pois para referência desta pesquisa um mínimo de três itens por fator foi adotada.

Após o descarte dos itens da dimensão Escola realizou-se a segunda tentativa gerando nova matriz de correlação e rodou-se novamente as medidas de adequabilidade para a verificação do novo modelo da dimensão Escola. O teste KMO foi de 0,73 e o teste de Esfericidade de Bartlett $\left(\mathrm{X}^{2}=547,75\right.$; df = 36; $\mathrm{p}=0,000)$. Novamente os dados se confirmaram como adequados para nova realização da AFE.

$\mathrm{Na}$ sequência os valores das comunalidades dos itens pelo método de extração de componentes principais foram: $1(0,55), 2(0,48), 3(0,40), 5$ $(0,59), 6(0,44), 7(0,61), 8(0,55), 11(0,53), 12$ $(0,52)$. Observou-se que os nove foram habilitados para a próxima etapa da AFE, pois atingiram patamar $\geq 0,40$.

Extraiu-se os fatores por meio da regra do autovalor $\geq 1$ (eigenvalue), conforme pode ser observado TABELA 1. 
Escala de apoio escolar e parental na formação esportiva

TABELA 1 -Total de variância explicada e número de fatores extraídos dimensão Escola - $2^{\mathrm{a}}$. tentativa

\begin{tabular}{ccccccc}
\hline \multirow{2}{*}{ Fatores } & \multicolumn{3}{c}{ Autovalores iniciais } & \multicolumn{2}{c}{ Somas rotacionadas das cargas ao quadrado } \\
\cline { 2 - 6 } & Total & \% da Variância & \% Acumulada & Total & \% da Variância & \% Acumulada \\
\hline 1 & 2,668 & 29,648 & 29,648 & 2,360 & 26,219 & 26,219 \\
2 & 1,990 & 22,114 & 51,762 & 2,299 & 25,543 & 51,762 \\
3 & 0,876 & 9,734 & 61,496 & & & \\
4 & 0,760 & 8,441 & 69,937 & & & \\
5 & 0,661 & 7,345 & 77,282 & & \\
6 & 0,609 & 6,770 & 84,052 & & \\
7 & 0,564 & 6,265 & 90,317 & & \\
8 & 0,471 & 5,232 & 95,549 & & \\
9 & 0,401 & 4,451 & 100,000 & & & \\
\hline
\end{tabular}

Visualiza-se um modelo fatorial com dois fatores extraídos, a partir da regra eigenvalue, adequados com variância explicada de $51,76 \%$ na dimensão Escola.

A FIGURA 1 apresenta o diagrama de inclinação (scree plot) e traz a representação da linha diagonal e horizontal onde pode ser observada a extração dos fatores da dimensão Escola.
Observa-se que a partir do fator dois a curva apresenta uma inclinaçáo acentuada (inflexão ou cotovelo) e no fator três uma cauda quase horizontal, portanto assume-se que o modelo fatorial de dois fatores seria o mais adequado para explicar os itens analisados.

As cargas fatoriais dos itens do modelo com nove itens são apresentadas na TABELA 2 a seguir.

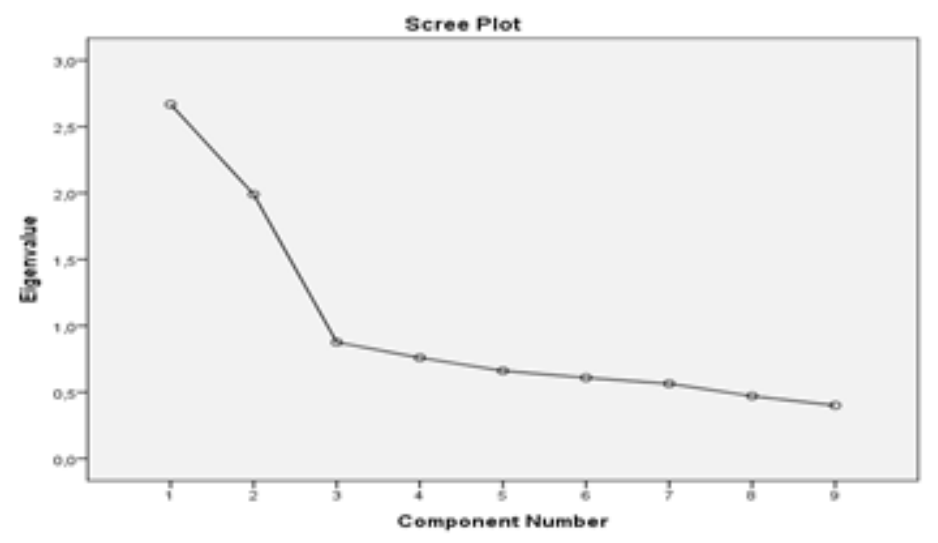

Fonte: Dados da Pesquisa.

FIGURA 1 -Diagrama de inclinação para itens dimensão Escola.

TABELA 2 -Estimação de fatores pelo Método de Extração de Componentes Principais e Rotação Varimax com Normalização Kaiser dimensão Escola $-2^{\mathrm{a}}$. tentativa

\begin{tabular}{ccc}
\hline Item & \multicolumn{2}{c}{ Fator } \\
\cline { 2 - 3 } & $\mathbf{1}$ & $\mathbf{2}$ \\
\hline 1 & 0,703 & 0,235 \\
2 & 0,691 & 0,051 \\
3 & 0,620 & 0,063 \\
5 & 0,764 & 0,097 \\
6 & 0,632 & $-0,198$ \\
7 & 0,101 & 0,776 \\
\hline
\end{tabular}

continua 


\begin{tabular}{ccc}
\hline & \multicolumn{2}{c}{ Fator } \\
\cline { 2 - 3 } Item & $\mathbf{1}$ & $\mathbf{2}$ \\
\hline 8 & 0,058 & 0,736 \\
11 & $-0,030$ & 0,730 \\
12 & 0,071 & 0,716 \\
\hline
\end{tabular}

Observa-se que a dimensão Escola se constituiu pelos itens 1, 2, 3, 5 e 6 no fator 1 conforme as cargas fatoriais e o fator 2 constituídos pelos itens 7, 8, $11 \mathrm{e}$ 12. Denominou-se como desenvolvimento esportivo o fator 1 e como desempenho escolar o fator 2 .

No processo analítico da dimensão Família, gerou-se a matriz de correlação e a verificação da adequabilidade dos dados, realizando-se sete tentativas. $\mathrm{Na}$ primeira tentativa com 27 itens, o teste $\mathrm{KMO}$ foi de 0,79 e o teste de Esfericidade de Bartlett $\left(\mathrm{X}^{2}=2024,51, \mathrm{df}=351, \mathrm{p}=0,000\right)$ reforçando a adequação dos dados conforme critérios vigentes e confirmando-se assim, a adequação do modelo para a análise fatorial.
Em seguida determinou-se os valores das comunalidades dos itens e para o item de número 25 o valor ficou abaixo do ponto de corte e foi descartado. Desta forma 26 itens foram mantidos para a próxima etapa. Todo o processo de realização das medidas de adequabilidade e AFE foi realizado novamente até que os resultados da purificação apresentassem robustez para a manutenção do modelo final.

No sentido de não tornar a descrição do processo maçante, foram resumidas no QUADRO 1 as diversas tentativas de purificação da escala e os motivos que levaram a exclusão de itens na aplicação de tentativas sequenciais da AFE.

QUADRO 1 -Tentativas de construção do modelo dimensão Família segundo procedimento da aplicação da Análise Fatorial Exploratória

\begin{tabular}{|c|c|c|}
\hline Tentativa & Itens incluídos na análise & 26 \\
\hline Problemas encontrados & \multicolumn{2}{|c|}{$\begin{array}{l}\text { Os itens } 5,10 \text { e } 20 \text { apresentaram cargas fatoriais cruzadas (maiores que } 0,40 \text { em dois fatores) } \\
\text { e foram descartadas da análise. Os itens } 17,18 \text { e } 27 \text { carregaram menos de } 3 \text { itens por fator e } \\
\text { foram descartadas da análise. }\end{array}$} \\
\hline Tentativa & Itens incluídos na análise & 20 \\
\hline Problema encontrado & \multicolumn{2}{|c|}{ O item 26 foi descartado por apresentar comunalidade abaixo de 0,40 . } \\
\hline Tentativa & Itens incluídos na análise & 19 \\
\hline Problemas encontrados & \multicolumn{2}{|c|}{$\begin{array}{l}\text { O item } 6 \text { apresentou carga fatorial cruzada (maior que } 0,40 \mathrm{em} \text { dois fatores). Os itens } 14 \text {, } \\
15,22 \text { e } 23 \text { carregaram apenas dois itens no fator e foram descartados. }\end{array}$} \\
\hline Tentativa & Itens incluídos na análise & 14 \\
\hline Problema encontrado & \multicolumn{2}{|c|}{ O item 24 foi descartado por apresentar comunalidade abaixo de 0,40 . } \\
\hline Tentativa & Itens incluídos na análise & 13 \\
\hline Problema encontrado & \multicolumn{2}{|c|}{$\begin{array}{c}\text { O item } 4 \text { foi descartado por apresentar carga fatorial cruzada, ou seja, maior que } 0,40 \mathrm{em} \\
\text { dois fatores. }\end{array}$} \\
\hline
\end{tabular}

Após as tentativas realizadas 12 itens na dimensão Família foram mantidos para a realização da validação numa sétima tentativa.

Foi gerada nova matriz de correlação e realizada em seguida a verificação da adequabilidade dos dados. $\mathrm{Na}$ sétima tentativa com 12 itens, o teste KMO foi de 0,75 e o teste de Esfericidade de Bartlett $\left(X^{2}=\right.$ $791,70, \mathrm{df}=66, \mathrm{p}=0,000)$, demonstrando que os dados estavam adequados para a rodagem da AFE.

ATABELA 3 apresenta os dados das comunalidades do itens componentes da dimensão família, modelo 12 itens ( $7^{a}$. tentativa). 
TABELA 3-Comunalidades dos itens da dimensão Família - modelo 12 itens $-7^{\mathrm{a}}$. tentativa - Extraído pelo Método de Componentes Principais

\begin{tabular}{ccccccc}
\hline & \multicolumn{7}{c}{ Itens } \\
\hline Inicial & $\mathbf{1}$ & $\mathbf{2}$ & $\mathbf{3}$ & $\mathbf{7}$ & $\mathbf{8}$ & $\mathbf{9}$ \\
Extraçáo & 1,000 & 1,000 & 1,000 & 1,000 & 1,000 & 1,000 \\
& 0,682 & 0,730 & 0,711 & 0,541 & 0,645 & 0,681 \\
Inicial & $\mathbf{1 1}$ & $\mathbf{1 2}$ & $\mathbf{1 3}$ & $\mathbf{1 6}$ & $\mathbf{1 9}$ & $\mathbf{2 1}$ \\
Extraçáo & 1,000 & 1,000 & 1,000 & 1,000 & 1,000 & 1,000 \\
\hline
\end{tabular}

Todos os itens da dimensão Família tiveram comunalidades acima do ponto de corte, portanto foram mantidos para o próximo passo da AFE.

Na TABELA 4 da dimensão Família observa-se o modelo fatorial com fatores extraídos, a partir da regra eigenvalue e o percentual da variância explicada.

Observa-se que o modelo fatorial com quatro fatores extraídos, a partir da regra eigenvalue, é adequado explicando $62,81 \%$ da variância na dimensão Família. A FIGURA 2 apresenta o diagrama de inclinação(scree plot) e traz a representação da linha diagonal e horizontal de forma que pode ser observada a extração dos fatores da dimensão Família modelo 12 itens ( 7 a . tentativa).

TABELA 4 -Total de variância explicada e número de fatores extraídos da dimensão Família, modelo de 12 itens $-7^{\mathrm{a}}$. tentativa

\begin{tabular}{ccccccc}
\hline \multirow{2}{*}{ Fatores } & \multicolumn{3}{c}{ Autovalores iniciais } & \multicolumn{3}{c}{ Somas rotacionadas das cargas ao quadrado } \\
\cline { 2 - 7 } & Total & \% da Variância & \% Acumulada & Total & \% da Variância & \% Acumulada \\
\hline 1 & 3,138 & 26,152 & 26,152 & 2,114 & 17,617 & 17,617 \\
2 & 2,031 & 16,921 & 43,073 & 1,921 & 16,011 & 33,628 \\
3 & 1,267 & 10,558 & 53,631 & 1,876 & 15,634 & 49,262 \\
4 & 1,102 & 9,186 & 62,817 & 1,627 & 13,555 & $\mathbf{6 2 , 8 1 7}$ \\
5 & 0,743 & 6,195 & 69,012 & & & \\
6 & 0,719 & 5,995 & 75,007 & & & \\
7 & 0,667 & 5,560 & 80,566 & & & \\
8 & 0,587 & 4,888 & 85,455 & & & \\
9 & 0,537 & 4,476 & 89,930 & & & \\
10 & 0,436 & 3,632 & 93,562 & & & \\
11 & 0,414 & 3,452 & 97,014 & & & \\
12 & 0,358 & 2,986 & 100,000 & & & \\
\hline
\end{tabular}

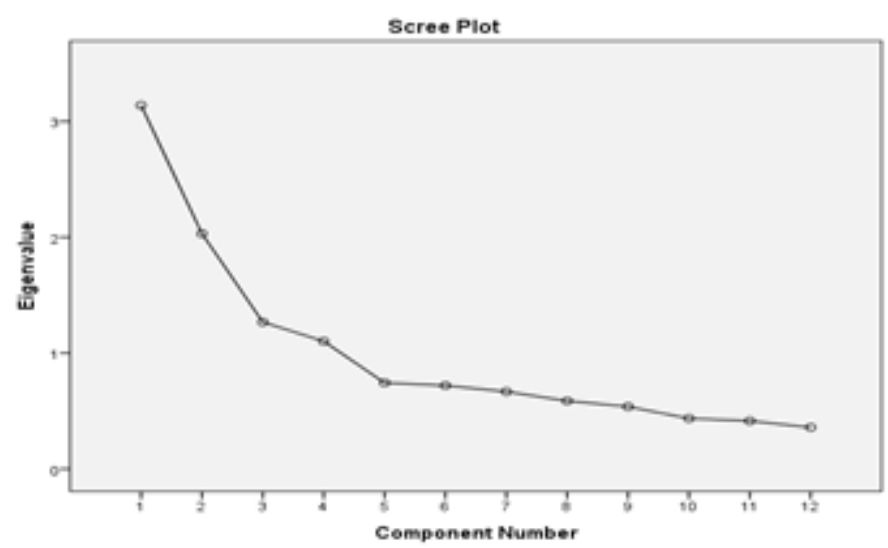

FIGURA 2 -Diagrama de inclinação para itens dimensão Família, modelo 12 itens ( $7^{\mathrm{a}}$. tentativa). 
Observa-se que a partir do fator quatro a curva apresenta uma inclinação acentuada (inflexão ou cotovelo) e no fator cinco uma cauda quase horizontal, portanto assume-se que o modelo fatorial de quatro fatores seria o mais adequado para explicar os itens analisados. As cargas fatoriais do modelo de 12 itens $-7^{\mathrm{a}}$. tentativa da dimensão Família são apresentadas na TABELA 5.

TABELA 5-Estimação de fatores pelo Método de Extração de Componentes Principais e Rotação Varimax com Normalização Kaiser dimensão Família modelo 12 itens $-7^{\mathrm{a}}$. tentativa

\begin{tabular}{ccccc}
\hline Item & \multicolumn{3}{c}{ Fator } & $\mathbf{4}$ \\
\cline { 2 - 5 } & $\mathbf{1}$ & $\mathbf{2}$ & $\mathbf{3}$ & 0,001 \\
$\mathrm{f} 1$ & 0,824 & 0,014 & 0,048 & 0,103 \\
$\mathrm{f} 2$ & 0,840 & 0,119 & 0,017 & 0,138 \\
$\mathrm{f} 3$ & 0,824 & $-0,106$ & 0,045 & 0,207 \\
$\mathrm{f} 7$ & 0,048 & $-0,012$ & 0,704 & 0,119 \\
$\mathrm{f} 8$ & 0,026 & 0,144 & 0,781 & 0,068 \\
$\mathrm{f} 9$ & 0,031 & 0,252 & 0,782 & 0,090 \\
$\mathrm{f} 11$ & 0,069 & 0,748 & 0,260 & 0,024 \\
$\mathrm{f} 12$ & 0,018 & 0,807 & 0,101 & 0,226 \\
$\mathrm{f} 13$ & $-0,054$ & 0,754 & 0,023 & 0,626 \\
$\mathrm{f} 16$ & 0,182 & 0,092 & 0,248 & 0,740 \\
$\mathrm{f} 19$ & $-0,006$ & 0,126 & 0,081 & 0,733 \\
$\mathrm{f} 21$ & 0,081 & 0,085 & 0,091 & \\
\hline
\end{tabular}

$\mathrm{Na}$ TABELA anterior as cargas fatoriais atendem aos pontos de corte, sendo assim a dimensão Família ficou constituída por: fator $1 \mathrm{com}$ os itens $1,2 \mathrm{e}$ 3, denominado de aspectos financeiros; fator 2 com os itens 11,12 e 13 nomeado de aspectos motivacionais; fator $3 \mathrm{com}$ os itens 7,8 e 9 denominado de interação esportiva e o fator 4 com os itens 16, 19 e 21 nomeado de acompanhamento esportivo.

Finalmente o modelo final do instrumento intitulado Contribuição da escola e da família na formação esportiva do adolescente (CEFFEA) constituiu-se de duas dimensóes, sendo uma Escola composta por dois fatores e outra Família composta por quatro fatores.

\section{Dimensáo Escola}

Fator 1 - desenvolvimento esportivo: No que concerne ao ambiente propício e infraestrutura esportiva adequada e com segurança que a escola oferta ao adolescente para o desenvolvimento no esporte.

Fator 2 - desempenho escolar: processo pelo qual o adolescente responde qualitativa e quantitativamente ao processo de aprendizado escolar, no qual a prática esportiva não interfere negativamente no seu desempenho escolar.

\section{Dimensão Família}

Fator 3 - aspectos financeiros: aportes necessários para a compra de materiais esportivos, insumos e contribuições para taxas e viagens com fins de intercâmbio e competiçôes.

Fator 4 - aspectos motivacionais: ações externas com o propósito de instigar o interesse em dedicar-se e investir energia em determinada tarefa.

Fator 5 - interação esportiva: ações em que a família demonstra ter interesse pela atividade esportiva praticada pelo adolescente ao procurar entender o estágio ao qual está submetido e estar em constante contato com a instituição.

Fator 6 - acompanhamento esportivo: açóes da família em participar do processo de formação esportiva de seus filhos, na qual demonstra interesse no esporte praticado pelo mesmo acompanhando tanto seus filhos nos eventos como o próprio esporte praticado por eles.

No QUADRO 2 a seguir, observa-se as dimensões do CEFFEA, com as respectivos fatores e os itens pertencentes aos mesmos. 
O instrumento é apresentado na FIGURA 3 na forma de numeração contínua, não idêntica ao questionário original, no intuito de facilitar o preenchimento por parte do adolescente.

QUADRO 2 - Divisão por dimensões, fatores e itens do CEFFEA

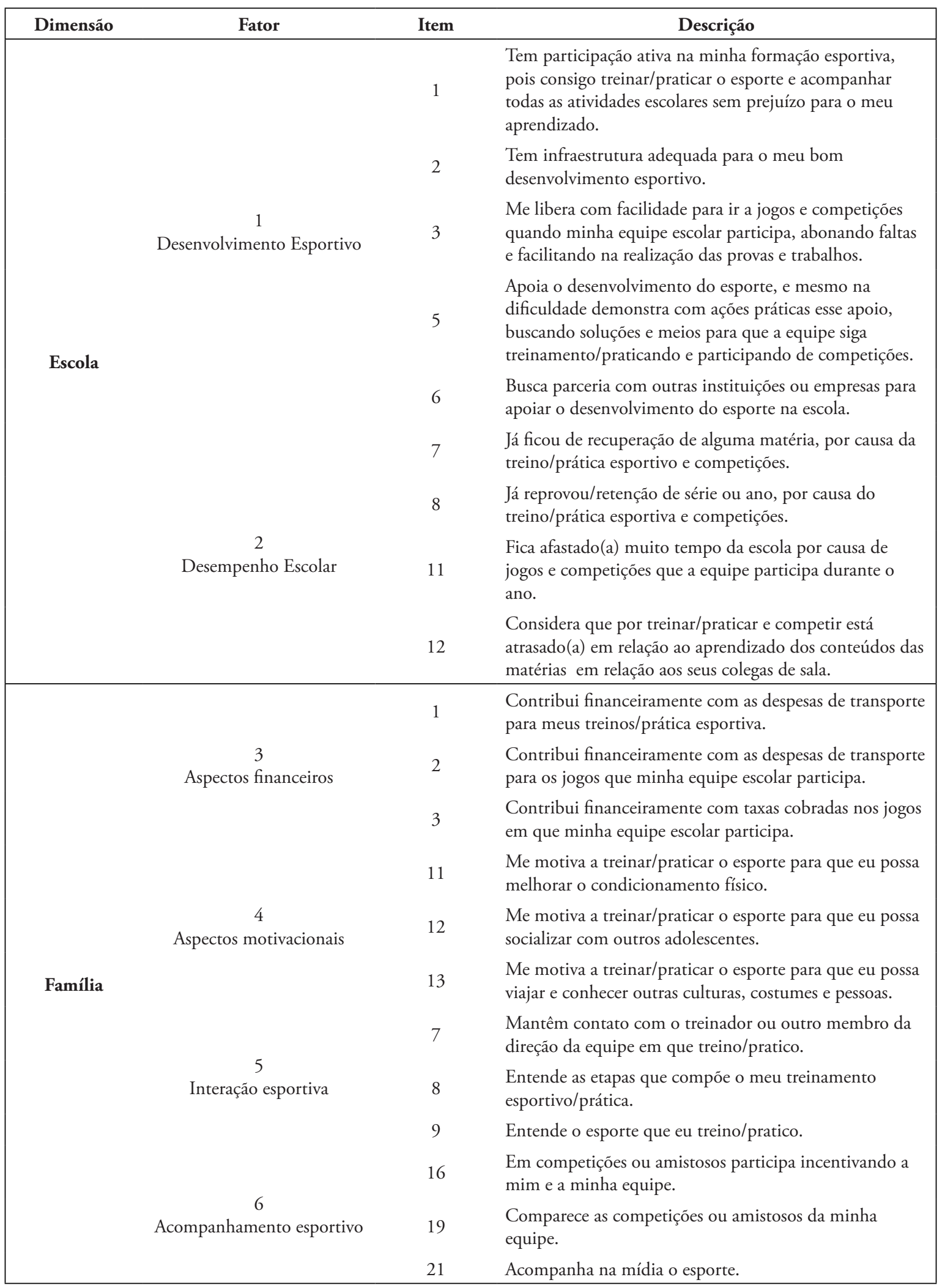




\section{QUESTIONÁRIO \\ CONTRUBUIÇĩo DA ESCOLA E DA FAMIUA NA FORMAÇÃo ESPORTIVA DO ADOLESCENTE}

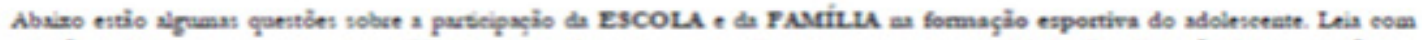

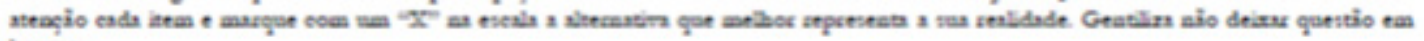
banee.

\section{MINHA FAMIILIA}

LEGENDA

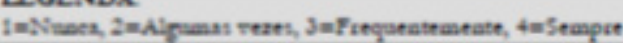

\begin{tabular}{|c|c|c|c|c|c|}
\hline 1 & 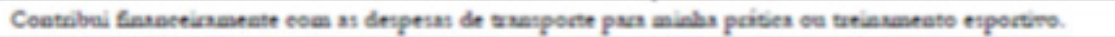 & 1 & 2 & 3 & 4 \\
\hline 2 & 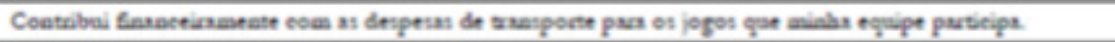 & 1 & 2 & 3 & 4 \\
\hline 3 & 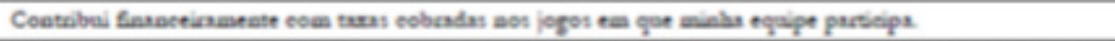 & 1 & 2 & 3 & 4 \\
\hline $\mathbf{s}$ & 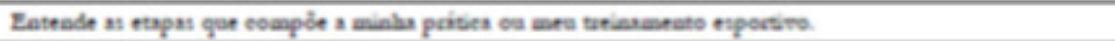 & 1 & 2 & 3 & 4 \\
\hline 6 & Entebde o eiporte qque tu partieo. & 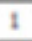 & 2 & 3 & 4 \\
\hline 8 & 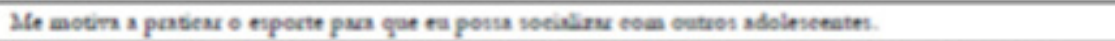 & 1 & 2 & 3 & 4 \\
\hline 9 & 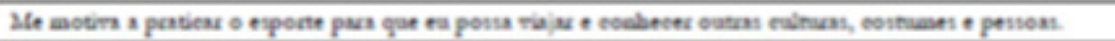 & 1 & 2 & 3 & 4 \\
\hline 10 & 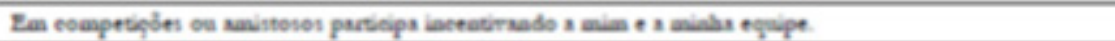 & $\overline{1}$ & 2 & 3 & 4 \\
\hline 11 & 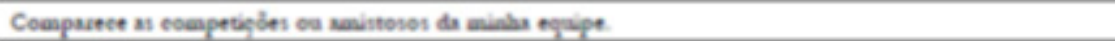 & $t$ & 2 & 3 & 4 \\
\hline
\end{tabular}

\section{MINHA ESCOLA}

LEGENDA

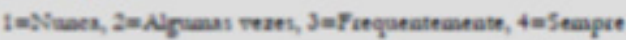

\begin{tabular}{|c|c|c|c|c|c|}
\hline 13 & 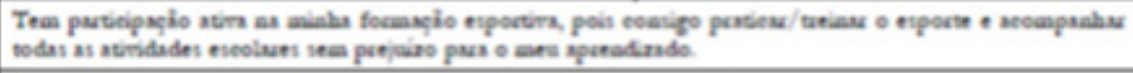 & $t$ & 2 & 3 & 4 \\
\hline 14 & 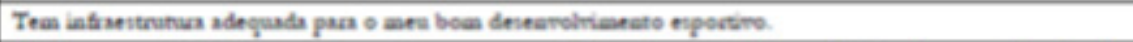 & $t$ & 2 & 3 & 4 \\
\hline 16 & 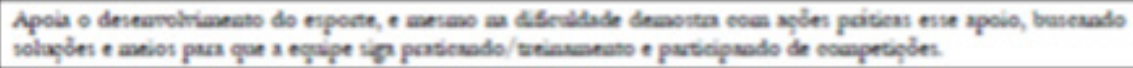 & $t$ & 2 & 3 & 4 \\
\hline
\end{tabular}

Para as questồes $15,19,20,21$ sesponda de acerdo coen a le zenda

\section{EM RELAÇĩo A SUA ESCOLA, VOCÊ}

\section{LEGENDA}

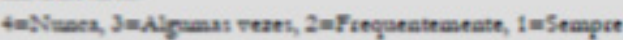

\begin{tabular}{|c|c|c|c|c|c|}
\hline 18 & 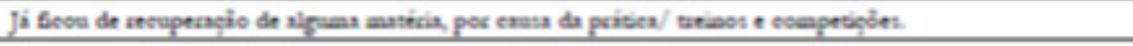 & 1 & 2 & 3 & 4 \\
\hline 20 & 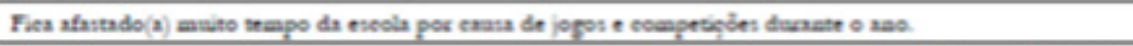 & 1 & 2 & 3 & 4 \\
\hline 21 & 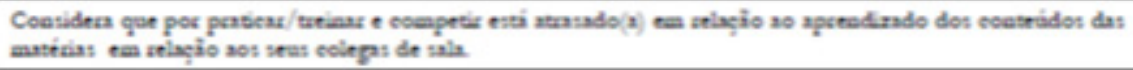 & 1 & 2 & 3 & 4 \\
\hline
\end{tabular}

FIGURA 3 -Questionário Contribuição da Escola e da família na formação esportiva do adolescente

\section{Discussão}

Após apuração dos resultados de confiabilidade (alfa de Conbrach) a dimensão escolar estabeleceuse com 11 itens $(\alpha=0,68)$, no entanto, essa perda não prejudicou a estrutura do questionário, conservando seu objetivo. A dimensão família $(\alpha$ $=0,79)$ não perdeu itens denotando-se assim que ambas as dimensões atingiram níveis aceitáveis de confiabilidade. A premissa de preservar ao menos um terço dos itens iniciais num processo de validação de instrumento ${ }^{29}$ foi alcançada no CEFFEA, corroborando com a literatura especializada ${ }^{41,42}$.

No que concerne ao eigenvalue os itens 9 e 10 da dimensão Escola apresentarem cargas fatoriais altas (0,81 e 0,87 respectivamente), no entanto foram os únicos a carregarem no fator 3. Desta forma não puderam ser confirmados nessa dimensão. Num 
contraponto encontra-se na literatura especializada, estudos que mantiveram esse status ${ }^{23,43}$. Porém em busca de maior robustez optou-se pela realização da purificação da escala ${ }^{44}$.

Após a purificação da escala, a dimensão Escola apresentou um pequeno aumento na adequabilidade dos dados, passando o KMO de 0,71 para 0,73 com o modelo de nove itens. Por outro lado o total da variância explicada teve ligeiro declínio de 56,38\% para $51,76 \%$. Essas alterações, no entanto, não mudaram o panorama da dimensão no estudo, uma vez que ficaram dentro dos mesmos patamares da primeira tentativa (antes da purificação da escala) e os pontos de cortes aceitáveis na literatura especializada ${ }^{34}$.

As cargas fatoriais finais do modelo de noves itens para dimensão Escola obtiveram valores bem acima dos níveis mínimos aceitáveis. Considerando a amostra inicial com 7,56 observações por item, na qual cargas fatoriais mínimas recomendadas na literatura seriam a partir de 0,40 , o resultado encontrado produziu uma variação de percentual acima da carga mínima de $50 \%$ a $90 \%$ acima desse valor mínimo, o que leva a entender que os itens carregados na dimensão Escola produzem robustez na dimensão e no instrumento como um todo.

Por outro lado pode-se encontrar no processo de validação de instrumento psicométrico, na fase analítica quando um item carregou carga fatorial dentro do limite mínimo recomendável, mas cujo fator não se confirmou por não apresentar número suficiente de itens, de forma subjetiva poder haver transferência do item para um fator próximo. A justificativa para tal procedimento é plausível, uma vez que no processo de validação de instrumentos psicométricos pode ocorrer o critério subjetivo. Para tanto o pesquisador deve perceber se o item de fato é importante para o instrumento ${ }^{36}$.

No que concerne a dimensão Família, após a realização da primeira tentativa, embora os dados de adequabilidade tenham sido aceitáveis, o item 25 "se sente pressionado(a) pela sua família a treinar/ praticar o esporte" atingiu nível abaixo do ponto de corte para a comunalidade $(0,36)$, desta forma é provável que a proporção da variância que é por ele explicada não foi aceitável na base de dados.

Observou-se a partir da segunda tentativa na dimensão Família que os itens: cinco "contribui com a minha alimentação visando meus treinamentos, práticas e jogos"; seis "contribui com despesas médicas quando necessito de algum tratamento por causa do meu treino/prática”; quatro "contribui com a compra de meu material esportivo: tênis, meia, uniforme de treino/prática, dentre outros"; 10 "me motiva a treinar/praticar o esporte para que eu possa ser um atleta profissional e ganhar dinheiro" e 20 "comenta sobre o jogo ou o resultado em que minha equipe participou" apresentaram cargas cruzadas, ou seja, carregaram carga maior que 0,40 em mais de um fator. Neste caso, a recomendação da literatura especializada é que se exclua o item. Essa recomendação se baseia no princípio de que a análise fatorial tem como um dos pressupostos a estrutura simples de seus componentes. Desta forma, uma variável não pode contribuir para a construção de fatores diferentes ${ }^{34}$. Ademais, um dos aspectos pode levar a esse problema são os erros de interpretação do item pelo respondente, provocando viés na resposta, concomitantemente provocando uma variabilidade dos dados, resultando numa correlação interitens semelhantes nos diferentes fatores de um construto $^{36}$. Buscando a robustez do instrumento os itens foram excluídos da dimensão.

Os itens 14 "me motiva a treinar/praticar o esporte para ajudar no pagamento do meu estudo"; 15 "me motiva a treinar/praticar o esporte para que eu ganhe bolsa de estudo numa universidade particular"; 22 "considera que o contato que sua família faz com membros da direção da sua escola contribui de forma positiva para o seu treino/prática esportiva" e 23 "considera que a contribuição da sua família no seu treino/prática esportiva é fundamental para o seu sucesso esportivo" carregaram em dois fatores que apresentaram apenas dois itens no fator embora as cargas 0,$77 ; 0,81 ; 0,79$ e 0,77 respectivamente fossem altas. Os itens 17 "em competiçôes ou amistosos chega a criticar a mim e a minha equipe", 18 "em competiçôes ou amistosos chegar a criticar o técnico da minha equipe" e 27 "conseguiria continuar treinando/praticando/competindo sem o apoio de sua família" carregaram item em um fator isolado, mesmo com cargas fatoriais altas $(0,77$; 0,$81 ; 0,81$ respectivamente), assim como ocorreu na dimensão Escola, os itens foram excluídos da dimensão, provocando nova tentativa. Há que se ressaltar que há sugestão na literatura que fatores com menos de três itens são incapazes de explicar o fator e pode diminuir a robustez de um instrumento ${ }^{34}$. No entanto, preconiza-se que, se optar por manter fator com menos de três itens, estes devem estar altamente correlacionados entre si $(r>0,70)$ e que tenham baixa correlação com os outros fatores ${ }^{45}$. No caso específico deste estudo na dimensão Escola os itens 14 e 15 e 22 e 23 essa 
premissa não ocorreu.

$\mathrm{Na}$ sétima tentativa da dimensão Família submeteu-se 12 itens aos procedimentos analíticos. As medidas de adequabilidade foram aceitáveis assim como as comunalidades. No processo de eigenvalue foram extraídos quatro fatores com o auxílio gráfico do scree plot. Esses fatores atingiram patamar de $62,8 \%$ de variância explicada. $\mathrm{Na}$ confirmação das cargas fatoriais, os itens carregaram nos fatores cargas acima do ponto de corte, variando entre $50 \%$ a $100 \%$, denotando que a dimensão Família contribui para a robustez do instrumento.

Embora o processo para a construção e validação de instrumentos psicométricos tenha critérios e rigor científico na busca por robustez ${ }^{29}$, há que se levar em consideração que um questionário com traço latente (construto) é subjetivo, não se descartando a hipótese de ocorrer viés no momento do indivíduo responder. No caso específico desta pesquisa o instrumento foi auto-administrado, voltado para um público específico (adolescentes) sem o auxílio do aplicador. Não descarta-se que a perda de itens possa ter ocorrido pela falta de atenção, que pode ter causado viés na resposta do adolescente, e detectado pelo processo analítico, mesmo que cuidados recomendados foram tomados para evitá-los.

Inicialmente foram previstos para a dimensão escola os fatores infraestrutura, desempenho escolar, suporte pedagógico, incentivo, reconhecimento e motivação para o esporte. Para a dimensão família os fatores foram financeiro, motivacional, educacional, relação familiar, amotivação, saúde, interação esportiva e acompanhamento esportivo. Porém, com o processo de validação, o modelo final foi confirmado por seis fatores, sendo dois para a dimensão escola (desenvolvimento esportivo e desempenho escolar) e quatro para a dimensão família (aspectos financeiros, aspectos motivacionais, interação esportiva e acompanhamento esportivo).

Após a aplicação da análise fatorial exploratória confirmou-se para as duas dimensões, fatores que englobaram itens que verificam o construto apoio, no entanto, além da perda de alguns itens como discutido anteriormente, não foram confirmados também alguns fatores previstos inicialmente para as duas dimensões. Buscando a robustez do CEFFEA optou-se por extrair os fatores amotivação da dimensão família e reconhecimento da dimensão escolar, pois os mesmos continham itens com cargas altas, no entanto não atingiram a correlação entre si $(r>0,70)$. Embora fosse importante para a compreensão do apoio escolar e parental na formação esportiva do adolescente, essa extração não prejudicou essa verificação no instrumento como um todo.

Os fatores iniciais da dimensão escola infraestrutura, suporte pedagógico e incentivo para o esporte foram agrupados em um único fator denominado de desenvolvimento esportivo, pois seus itens foram agrupados no fator um pelos procedimentos analíticos. Já o fator motivação não carregou itens suficientes para se manter. No entanto, o fator desempenho escolar se confirmou por meio da AFE.

Os fatores iniciais da dimensão família, educacional e relação familiar não se mantiveram por não carregar itens suficientes após a AFE. Já o fator inicial saúde não se confirmou, no entanto teve item carregado no fator quatro nomeado de aspecto motivacional que se confirmou. Os fatores iniciais aspectos financeiros, interação esportiva e acompanhamento esportivo se confirmaram através dos procedimentos empírico e analítico. É comum que estas responsabilidades sejam atribuídas a família, independentemente da classe econômica, corroborando assim esse resultado com outros estudos na literatura especializada $a^{2,10-15}$.

Foi carregado no questionário apenas um item com característica de apoio dual escola-família, o fator 5 "Mantêm contato com o treinador ou outro membro da direção da equipe em que treino/pratico". Porém, a parceria entre essas duas dimensões é relevante na formação esportiva do adolescente e é necessária uma relação mais próxima entre elas, um maior número de itens poderia contemplar essa dualidade.

Todas as medidas de adequabilidade dos dados e dos modelos fatoriais ou soluções realizadas apresentaram níveis satisfatórios, desta forma, pode-se concluir que o presente questionário (CEFFEA) é um instrumento que contém validação teórica, fidedignidade, adequabilidade e evidências de validade de construto, se propondo verificar o apoio da escola e da família na formação esportiva do adolescente.

$\mathrm{O}$ instrumento apresenta evidências de validade de construto, porém não apresenta evidências de validade externa. A dificuldade de encontrar outros questionários confiáveis e similares na língua portuguesa e elevação da duração do tempo para participar da pesquisa foram fatores considerados para aceitar esta limitação.

Sugere-se para estudos futuros a inclusão de outros tipos de validade como a externa, que sejam inclusos 
itens que possam unir as duas dimensões (escola e família) dando maior robustez a contribuição dual na formação esportiva do adolescente. Esse acompanhamento mútuo refletirá positivamente na sua formação esportiva, pois são duas dimensões de convivência contínua nesse período de sua vida.
A aplicação do instrumento numa amostra maior para que sejam gerados dados estatísticos para a construção de uma tabela de normatização, com $\mathrm{n}$ da amostra adequado para este fim conforme literatura vigente e para o melhoramento do modelo apresentado.

\title{
Agradecimentos
}

Agradecemos o apoio da Secretaria de Estado de Educação, Secretaria de Estado de Esporte de Mato Grosso e a Secretaria de Esportes de Tangará da Serra-MT.

\begin{abstract}
Development and validation of a school and parental support tool in sports training adolescent

The purpose of this study was to construct and validate a survey to ascertain the school and family's contribution in adolescents sports formation. The participants were 295 adolescents who practice in team and individual sports. It was carried out the following steps to validate the instrument: theoretical validation, which was the instrument of the construction and the pilot questionnaire implementation; and analytical empirical validation, which consisted of the questionnaire application, verification of the reliability, verification of suitability, application of the exploratory factor analysis and modeling end of the questionnaire. The reliability analysis, suitability and factorial exploratory performed to identify evidence of construct validity of the questionnaire showed acceptable adjustments and results that met the literature recommendations. The final model of the questionnaire items covered 21 of the 39 analyzed earlier and were divided into two dimensions: School with nine items divided into so-called factors of (1) sports development (items 1, 2, 3, 5 and 6) and (2) school performance (items 7, 8, 11 and 12); and Family with 12 items divided into so-called factors of (1) financial aspects (items 1, 2 and 3), (2) motivational aspects (items 11, 12 and 13), (3) Sports Interaction (7, 8 and 9) and (4) Sports tracking (items 16, 19 and 21). It is concluded that the questionnaire showed satisfactory levels of reliability and internal consistency with evidence of construct validity for checking of the support that the school and the family are concentrating on sporting formation of adolescents.
\end{abstract}

KEYWORDS: Validation; Sport; Adolescent; School Support; Parental Support.

\section{Referências}

1. Hohmann A, Seidel L. Scientific aspects of talento development. Int J Physical education. 2003;1:9-20.

2. Bloom BS. Developing talento in Young people. New York: Ballantine Books; 1985.

3. Weiss MR. Coaching children to embrace a love of the game. Olympic Coach. 2004;16:153-177.

4. Stevens A, Schmidt MI, Duncan BB. Desigualdades de gênero na mortalidade por doenças crônicas não transmissíveis no Brasil. Ciênc Saúde Coletiva. 2012;17:2627-2634.

5. Hillmann $\mathrm{CH}$, Castelli DM, Buck SM. Aerobic fitness and neurocognitive function in healthy preadolescent children. Med Sci Sports Exerc. 2005;37:1967-1974.

6. Tammelin T, Näyhä S, Hills AP, Järvelin MR. Adolescent participation in sports and adult physical activity. Am J Prev Med. 2003;24:22-28. 
7. Tavares BF, Beria JU, Lima MS. Prevalência do uso de drogas e desempenho escolar entre adolescentes. Rev Saúde Pública. 2001;35:150-158.

8. Starepravo FA, Mezzadri FM. Esporte, relaçóes sociais e violências. Motriz: Rev Educ Fís. 2003;9:49-52.

9. Gonzáles MJM. O deporte espectáculo como elemento civilizador dende a perspectiva dos espectadores deportivos: unha análise dende a filosofía da "nonviolencia" e a teoría dos ámbitos intercondicionantes. Conexôes. 2000;4:117128.

10. Kirk D, Carllson T, O’Connor A, Burke P, Davis K, Glover S. The economic impact on families of children's participation in junior sport. Aust J Sci Med Sport. 1997;29:27-33.

11. Coakley J. Sports in society: issues and controversies. Saint Louis: Times Mirror/Mosby College; 2008.

12. Côté J, Wimmer C, Beamer M. The role of the family in developing talent in figure skating. Ottawa: Canadian Figure Skating Association; 1998.

13. Ferreira RM, Moraes LC. Influência da família na primeira fase de desenvolvimento da carreira de nadadores medalhistas olímpicos brasileiros. Motri. 2012;8:42-51.

14. Nunomura M, Oliveira MS. A participação dos pais na carreira das atletas femininas de ginástica artística: a perspectiva dos técnicos. Rev Bras Educ Fís Esporte. 2014;1:125-134.

15. Reis CP, Ferreira MCC, Moraes LCCA. O apoio dos pais ao desenvolvimento da carreira de atletas masculinos de basquetebol. Rev Bras Ciênc Esporte. 2016;38(2):149-155. doi: http://dx.doi.org/10.1016/j.rbce.2015.10.007.

16. Vissoci JRN, Oliveira LP, Nascimento Junior JRA. A influência do suporte parental no desenvolvimento atlético de jogadoras de futsal. Rev Psicol: Teor Prát. 2013;15:145-156.

17. Vieira LF, Mizoguchi MV, Garcia Junior E, Garcia WF. Estilos parentais e motivação em atletas jovens de futebol de campo. Pensar Prát. 2013;16:13-19.

18. Timperio AF, van Stralen MM, Brug J, Bere E, Chinapaw MJM, de Bourdeaudhuij I, et al. Direct and indirect associations between the family physical activity environment and sports participation among 10-12 year-old european children: testing the EnRG framework in the ENERGY project. Int J Behav Nutr Phys. 2013;10:1-10.

19. Silva PVC, Fleith DS. Atletas talentosos e o papel desempenhado por suas famílias. Rev Bras Psicol Esp. 2010;3:42-63.

20. Kobarg APR, Vieira V, Vieira ML. Validação da escala de lembranças sobre práticas parentais - EMBU. Aval Psicol. 2010;9:77-85.

21. Howle TC, Whipp PR, Gainrs F, Thornton AL, Rebar AL, Jackson B. Construct validity evidence for a measure of peer athletic reputation (PathR) in high school physical education. Psychol Sport Exerc. 2016;24:9-17.

22. Nascimento Júnior JRA, Vissocci JRN, Lavallee D, Vieira LF. Adaptation and validation of the Sport Multidimensional Perfectionism Scale-2 (SMPS-2) for the Brazilian sport context. Motriz: Rev Educ Fís. 2015;2:125-136.

23. Gomes A, Zão D. Envolvimento parental e orientação motivacional na prática desportiva: desenvolvimento de instrumentos de avaliação e análise das percepçóes de pais e atletas. Psicol: Teor Inv Prátic. 2007;2:319-339.

24. Moraes LC, Rabelo AS, Salmela JH. Papel dos pais no desenvolvimento de jovens futebolistas. Psicol Reflex Crític. 2004;17:211-222.

25. Domingues MP, Cavichiolli F, Gonçalves CE. Perspectiva ecológica na determinação de percursos desportivos contrastantes em jovens futebolistas. Rev Bras Educ Fís Esporte. 2014;2:249-261.

26. Aroni AL. Motivação e percepção do envolvimento parental na prática desportiva de jovens nadadores [dissertação]. Lisboa (PT): Universidade Lusófona de Humanidades e Tecnologia, Faculdade de Educação Física e Desporto; 2011.

27. Luguetti CN, Bastos FC, Böhme MTS. Gestão de práticas esportivas escolares no ensino fundamental no município de Santos. Rev Bras Educ Fís Esporte. 2011;25:237-49.

28. Greco PJ. O sistema de formaçáo e treinamento esportivo do goleiro de handebol. In: Greco PJ, organizador. Caderno de rendimento do atleta de handebol. Belo Horizonte: Health; 2000. p.21-32.

29. Pasquali L. Testes referentes a construto: teoria do modelo de construção. In: Pasquali, L, organizador. Instrumentos Psicológicos: Manual prático de elaboração. Brasília: LabPAM, IBAPP; 1999. p.37-71.

30. Pasquali L. Psicometria. Rev Esc Enferm. 2009;43:992-999.

31. Maroco J, Garcia-Marques T. Qual a fiabilidade do alfa de Cronbach? Questôes antigas e soluções modernas? Laboratório Psicologia. 2006;1:65-90.

32. Matthiensen A. Uso do Coeficiente Alfa de Cronbach em Avaliaçóes por Questionários. Boa Vista: Embrapa Roraima; 2011.

33. Matos DAS. Confiabilidade e concordância entre juízes: aplicaçôes na área educacional. Est Aval Educ. 2014;25:298324. 
34. Hair JF, William B, Babin B, Anderson RE. Análise multivariada de dados. Porto Alegre: Bookman; 2009.

35. Figueiredo DB, Silva JA. Visão além do alcance: uma introdução à análise fatorial. Opin Pública. 2010;16:160-185.

36. Faccenda O, Dalben A, Freitas LC. Capacidade explicativa de questionários de contexto: aspectos metodológicos. Rev Bras Est Pedag. 2011;92:246-267.

37. Pallant J. SPSS Survival Manual. Buckinghan: Open University Press; 2007.

38. Dancey CP, Reidy J. Estatística sem matemática para psicologia. Porto Alegre: Artmed; 2006.

39. Associação Brasileira de Empresas de Pesquisa. Critério classificação econômica Brasil. São Paulo (SP): ABEP; 2015.

40. Stemler SE. A comparison of consensus, consistency, and measurement approaches to estimating interrater reliability. PARE. 2004;9:1-19.

41. Greco PJ, Morales JCP, Aburachid LMC, Silva SR. Evidência de validade do teste de conhecimento tático processual para orientação esportiva TCTP: OE. Rev Bras Educ Fís Esporte. 2015;29:313-324.

42. Aburachid LMC, Greco PJ. Processos de validação de um teste de conhecimento tático declarativo no tênis. Rev Educ Fís/UEM. 2010;21:603-610.

43. Zequinão MA, Cardoso FL. Criação e avaliação das qualidades psicométricas do questionário de satisfação dos clientes em serviços de educação física (QSCSEF). Rev Bras Ciênc Esporte. 2013;35:481-501.

44. Parassuraman V. SERVQUAL: A multiple-item scale for measuring consumer perceptions of service quality. J Retailing. 1989;64:12-40.

45. Tabachnick BG, Fidell LS. Using multivariate statistics. New York: Harper and Row; 2001.

ENDEREÇO DE CORRESPONDÊNCIA:

Erasmo Braz dos Santos

Rua Manuel Bandeira, Quadra 52, Lote 11 - Santa Cruz I

Cuiabá - MT - BRASIL CEP: $78068-290$

E-mail: erasmobraz@bol.com.br; erasbraz@gmail.com
Submetido: 13/04/2016

Revisado: 17/06/2016

Aceito: 10/08/2017 